\title{
Differences among skeletal muscle mass indices derived from height-, weight-, and body mass index-adjusted models in assessing sarcopenia
}

\author{
Kyoung Min Kim, Hak Chul Jang, and Soo Lim
}

Department of Internal Medicine, Seoul National University College of Medicine and Seoul National University Bundang Hospital, Seongnam, Korea

Received: January 25, 2016 Accepted: May 15, 2016

\section{Correspondence to}

Soo Lim, M.D.

Department of Internal Medicine, Seoul National University College of Medicine and Seoul National University Bundang Hospital, 82 Gumi-ro 173beon-gil, Bundang-gu, Seongnam 13620, Korea

Tel: +82-31-787-7035

Fax: $+82-31-787-4051$

E-mail:limsoo@snu.ac.kr
Aging processes are inevitably accompanied by structural and functional changes in vital organs. Skeletal muscle, which accounts for $40 \%$ of total body weight, deteriorates quantitatively and qualitatively with aging. Skeletal muscle is known to play diverse crucial physical and metabolic roles in humans. Sarcopenia is a condition characterized by significant loss of muscle mass and strength. It is related to subsequent frailty and instability in the elderly population. Because muscle tissue is involved in multiple functions, sarcopenia is closely related to various adverse health outcomes. Along with increasing recognition of the clinical importance of sarcopenia, several international study groups have recently released their consensus on the definition and diagnosis of sarcopenia. In practical terms, various skeletal muscle mass indices have been suggested for assessing sarcopenia: appendicular skeletal muscle mass adjusted for height squared, weight, or body mass index. A different prevalence and different clinical implications of sarcopenia are highlighted by each definition. The discordances among these indices have emerged as an issue in defining sarcopenia, and a unifying definition for sarcopenia has not yet been attained. This review aims to compare these three operational definitions and to introduce an optimal skeletal muscle mass index that reflects the clinical implications of sarcopenia from a metabolic perspective.

Keywords: Sarcopenia; Muscle mass; Muscle mass index; Frailty; Appendicular skeletal muscle mass

\section{INTRODUCTION}

Muscle tissue has diverse functions, including maintaining body posture and producing mobility and movement in cooperation with the skeleton [1]. It also significantly contributes to energy metabolism $[1,2]$. Aging processes are inevitably accompanied by weakened structure and attenuation of the functions in many organs. Muscle tissue also deteriorates with aging both quantitatively and qualitatively $[3,4]$.

Sarcopenia refers to a syndrome characterized by se- rious loss of skeletal muscle mass and strength $[5,6]$. Because muscle tissue plays important functional and metabolic roles, sarcopenia threatens the health of elderly people, and it is accompanied by many adverse outcomes $[7,8]$. Because skeletal muscle tissue supports the mobility of bone and also contributes to bone strength, sarcopenia increases the risks of falls, osteoporosis, and fractures [9]. Muscle tissue also contributes to energy metabolism [10]. Thus, sarcopenia is closely related with metabolic derangements and may be associated with the occurrence of cardiovascular diseases in the elderly 
[2,11-13]. With the rapid increase in the number of elderly people, sarcopenia exerts a growing impact on health status in the population.

Recently, many study groups have suggested operational diagnostic criteria for sarcopenia $[5,14]$. Sarcopenia is commonly defined by a combination of low muscle mass with low muscle strength or impaired performance $[14,15]$. Hand grip strength and leg extension/ flexion strength have both been used as indices of muscle strength [16]. Gait speed has been used for muscle performance evaluation [16]. Although muscle mass is not specifically a predictor of muscle strength or physical performance, it is significantly correlated with these parameters. Low muscle mass potentially contributes to disability and frailty in the elderly [17].

Therefore, the accurate measurement of muscle mass is a crucial step for classifying sarcopenic subjects, who have higher risks of adverse events related to frailty. In assessing muscle mass, dual-energy X-ray absorptiometry (DXA) or bioelectrical impedance analysis (BIA) methods are the most widely used tools. Several equational methods have been proposed to quantify the relative muscle mass from the absolute values of regional muscle masses [17-19]. In this context, study groups from different regions have released statements about the definition and assessment of sarcopenia. They have all recommended one of the muscle mass indices among several suggested methods $[5,14,17,20]$. However, the prevalence and clinical implications of sarcopenia differ according to the methods used for determining low muscle mass $[21,22]$. This review aims to compare the various skeletal muscle mass indices and to introduce representative skeletal muscle mass indices for use in the Korean population.

\section{SKELETAL MUSCLE MASS INDEX FOR DEFIN- ING SARCOPENIA}

\section{Quantification and operational definitions of skele- tal muscle mass index}

Measuring the amount of muscle mass is the basis of assessing sarcopenia [14]. Several techniques can be used to quantify muscle mass including DXA, BIA, computed tomography, and magnetic resonance imaging. Among them, DXA and BIA are the most widely used modali- ties because they are less expensive, lower risk, and more available than the others. DXA provides absolute values for total muscle mass and regional muscle mass. It can also display the skeletal muscle mass index. BIA is a good alternative method for assessing muscle mass. Total muscle mass and appendicular skeletal muscle mass (ASM) can also be estimated by prediction equations with the BIA method $[23,24]$.

ASM, which is a sum of the muscle mass of both arms and legs, is generally used for the skeletal muscle mass index. However, muscle mass is fundamentally correlated with body size, indicating that subjects with a larger body size may have larger muscle mass [25]. Therefore, when evaluating the adequacy of muscle mass, the absolute level of ASM has been used after adjusting for body size in different ways, namely using height squared (ASM/ht ${ }^{2}$ ), weight (ASM/wt), or body mass index (ASM/ BMI) [17-19].

$\mathrm{ASM} / \mathrm{ht}^{2}$ was first suggested by Baumgartner et al. [18] in the New Mexico Elder Health Survey. This index provided significant associations with physical disability or frailty, although the associations were relatively weaker in women [18]. Since then, many research groups have used this index for defining sarcopenia, and some have reported associations between this index and many clinical outcomes [26-28]. However, because this index is positively correlated with BMI, it has the limitation that subjects with a greater BMI due to a larger amount of fat are less likely to be classified as having sarcopenia [29].

Another skeletal muscle index, which is adjusted for fat mass along with height, was suggested by Newman et al. [29]. Although this index partially overcame previous problems, it has not been used widely because it is not simple to calculate. Briefly, the equational methods to calculate ASM adequately were derived from linear regression analyses among ASM, height, and fat mass in the study populations [29]. The differences between the actual ASM values and predicted ASM levels were used to identify sarcopenic subjects [29].

In 2002, a weight adjusted muscle mass index was proposed by Janssen et al. [19]. Their skeletal muscle mass index calculated the total muscle mass adjusted for weight. Sarcopenia as defined by this skeletal muscle index was significantly related to functional impairment and disability [19]. This weight adjusted model was subsequently modified as ASM/wt. This index has been 
widely used as an alternative method together with the height squared-adjusted index described by Baumgartner et al. [30,31].

More recently, another muscle mass index, the ASM/ BMI index, was introduced by the Foundation for the National Institutes of Health (FNIH) Sarcopenia Project in 2014 [17]. Use of this index is growing [32,33].

\section{Recommended skeletal muscle mass index by inter- national consensus for sarcopenia}

The European Working Group on Sarcopenia in Older People (EWGSOP), International Working Group on Sarcopenia(IWGS), Asian Working Group for Sarcopenia (AWGS), and FNIH Sarcopenia Project have tried to establish consensus diagnostic criteria for sarcopenia, and they recently released practical guidelines [5,14,20,34]. These guidelines generally agreed on the mechanisms and clinical implications of sarcopenia in the elderly and, above all, on the fact that both muscle strength and muscle mass are important in the concept of sarcopenia. However, the skeletal muscle mass indices suggested in the respective guidelines differ somewhat. In 2010, the EWGSOP released their consensus on the definition and diagnosis of sarcopenia, which was agreed upon by four participant organizations, the European Union Geriatric Medicine Society, European Society of Clinical Nutrition and Metabolism, International Academy of Nutrition and Aging, and International Association of Gerontology and Geriatrics-European Region [14]. The EWGSOP recommended a diagnostic algorithm with a composite of attenuated muscle strength and low muscle mass. That consensus presented both ASM $/ \mathrm{ht}^{2}$ and weight adjusted total muscle mass for use when calculating muscle mass [14]. They also provided cutoff points for low muscle mass at two standard deviations (SDs) below the mean reference values of healthy young adults [14]. The IWGS released a consensus on sarcopenia in 2011 [5], recommending the use of the ASM/ht ${ }^{2}$ index for determining relative muscle mass. They also proposed whole-body lean mass adjusted for height squared as an alternative method. In regard to cutoff points for low muscle mass, they suggested different thresholds than the EWGSOP did, using the lowest quintile of values for healthy young adults [5]. A consensus report for Asian ethnicity was published in 2014 by the AWGS [20]. They also recommended using the $\mathrm{ASM} / \mathrm{ht}^{2}$ index to deter- mine muscle mass. In terms of cutoff values for sarcopenia, they proposed either two SDs below the mean levels of young healthy adults or the lowest quintile of ASM/ $\mathrm{ht}^{2}$ index [20].

Thus, the definitions and cutoff values for low muscle mass in several consensus reports differ among the working groups. Since these international statements about sarcopenia were released, the number of studies about sarcopenia has increased drastically. Studies have used various muscle mass indices, including ASM/ $\mathrm{ht}^{2}$, ASM/wt, or total muscle mass/wt [11,16,26,35,36]. Furthermore, the question of the discordance between two representative indices, $\mathrm{ASM} / \mathrm{ht}^{2}$ and $\mathrm{ASM} / \mathrm{wt}$, has been raised [21,30,37,38].

Recently, the FNIH Sarcopenia Project proposed the most recent consensus on the definition and operational criteria for sarcopenia based on large and diverse population-based studies $[15,17,34]$. They recommended ASM/BMI as a new operational method for calculating muscle mass [17]. They performed statistical classification and regression tree analysis to calculate the muscle mass index that is most strongly and directly correlated with weakness and slowness [17]. Only limited numbers of studies have validated the clinical relevance of this index in different study populations [32,33].

\section{Differences among skeletal muscle mass indices in regard to age trends and sarcopenia prevalence}

So far, several operational methods for defining low lean mass have been suggested, including ASM/ $\mathrm{ht}^{2}$, ASM/wt, and ASM/BMI. Among these parameters, the most appropriate method with the highest predictive value for identifying subjects who are at higher risk of weakness and slowness remains uncertain. Moreover, the prevalence of sarcopenia varies considerably depending on the method used [21,29,39,40].

Fig. 1 shows the trends of each muscle mass index with age using data from the Korea National Health and Nutrition Examination Survey (KNHANES) 2008 to 2010. In brief, KNHANES is a nationwide, population-based, cross-sectional study. It has been conducted periodically since 1998 by the Division of Chronic Disease Surveillance of the Korea Centers for Disease Control and Prevention to assess the health and nutritional status of the civilian, non-institutionalized population of Korea. Many clinical studies about sarcopenia in Ko- 
rea have been conducted using these national data. After excluding several chronic diseases that can affect muscle mass, a total of 28,476 men and women older than 10 years were included in analyses from the original data of KNHANES 2008 to 2010. Although there is an evident gender difference, in both men and women, total muscle mass and ASM show similar patterns with aging (Fig.

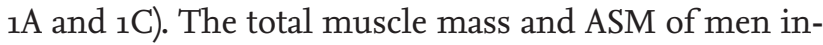
creased dramatically until their 30 and then decreased continuously until 90 years of age, with a slight acceleration after 60 years of age (Fig. 1A). However, changes in muscle index with aging happened quite differently in women, indicating that total muscle mass and ASM in women increased slowly until their 40s, remained constant until their 50 s to 6os, and then began to decrease (Fig. 1 C). As expected, the total amount of increment or decline in muscle mass change with aging was smaller in women than in men.

Three proposed operational methods for determining muscle mass to assess sarcopenia are presented in Fig. 1. These indices showed different trends between men and women. They were generally similar to each other and also similar to ASM or total muscle mass in men (Fig. 1B). All three indices increased until the 20 s to 30 s, and then decreased with aging. The ASM/wt index showed the least changes with aging among the three indices (Fig. 1B). However, the age patterns of these three indices developed differently in women. The ASM $/ \mathrm{ht}^{2}$ value increased continuously until the 6os, and then decreased slowly, whereas the ASM/wt value gradually decreased until the age of 60 years, followed by a trivial rise. Meanwhile, the pattern with aging in the ASM/BMI index was distinguishable from both the ASM/ $\mathrm{ht}^{2}$ and the ASM/wt indices, remaining relatively constant until the zos and then decreasing continuously (Fig. 1D). Based on these observations of the wide differences in patterns with aging using the three parameters, the prevalence of sarcopenia is likely to differ depending on the method used
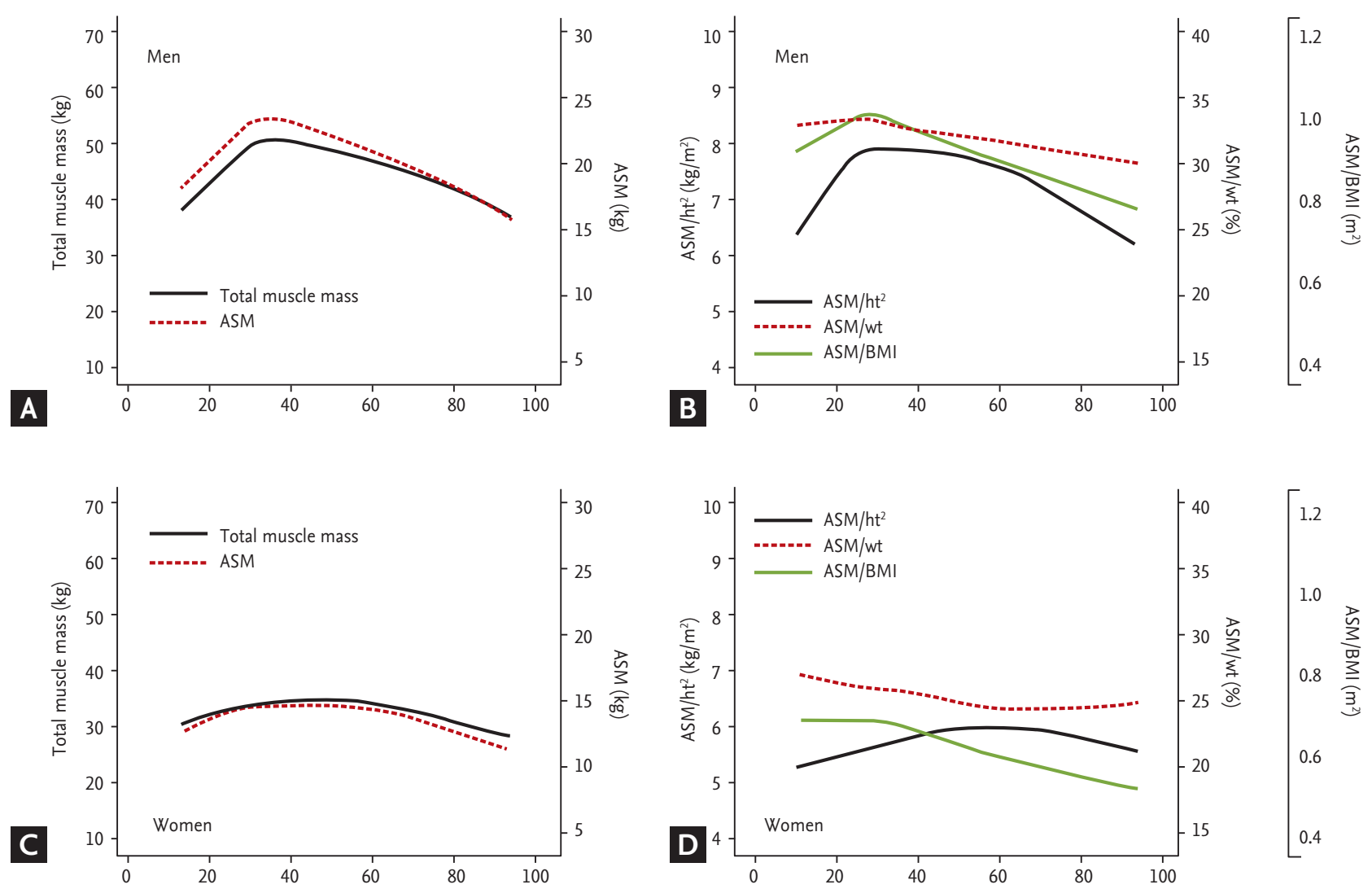

Figure 1. Age trends in skeletal muscle mass indices in (A, B) men and (C, D) women. ASM, appendicular skeletal muscle mass; $\mathrm{ht}^{2}$, height squared; wt, weight; BMI, body mass index. 
Normal Class | sarcopenia Class II sarcopenia
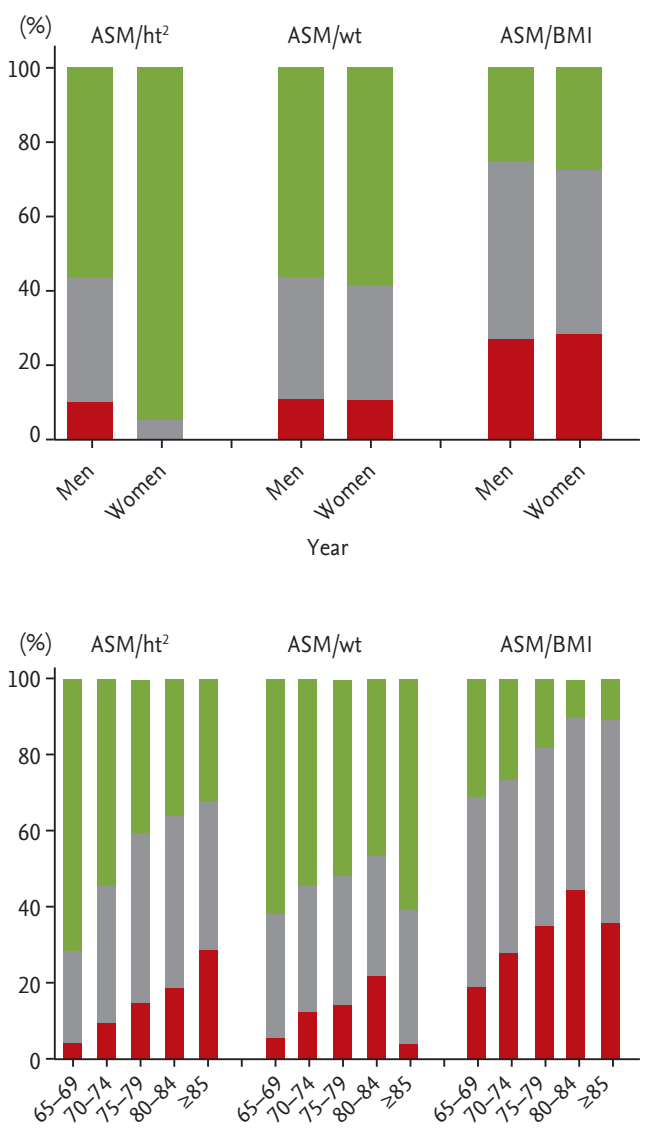

B

Year

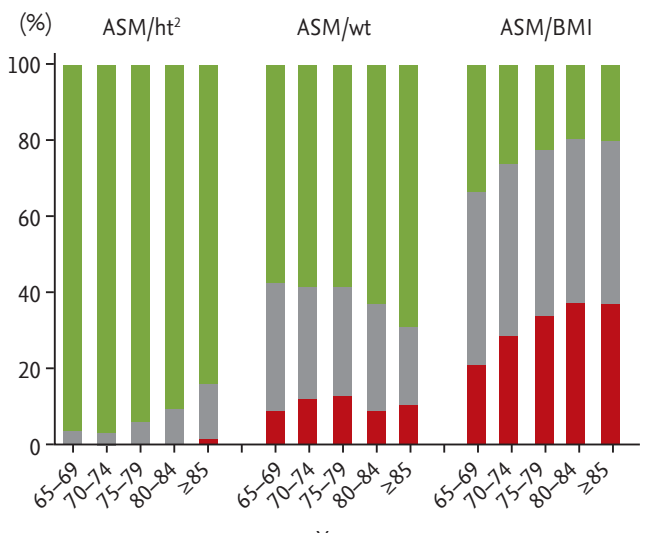

C

Year

Figure 2. Prevalence rates of sarcopenia in (A) men and women aged 65 years or older and in each age group stratified by 5-year intervals for (B) men and (C) women according to three different operational methods. Class I and class II sarcopenia were defined when their values were within -1.0 to -2.0 standard deviation (SD) or below $-2.0 \mathrm{SD}$ of the sex-specific mean of a young reference group (20 to 29 years old), respectively. ASM, appendicular skeletal muscle mass; $h \mathrm{t}^{2}$, height squared; wt, weight; BMI, body mass index. for defining low muscle mass.

Applying the three operational methods given above, the prevalence rates of sarcopenia based on low muscle mass were analyzed in the Korean elderly population aged 65 years and older (Fig. 2). Class I and class II sarcopenia were defined when their values were within -1.0 to $-2.0 \mathrm{SD}$ or below $-2.0 \mathrm{SD}$ of the sex-specific mean of a young reference group (20 to 29 years old), respectively, as previously proposed [19]. As shown in Fig. 2, the prevalence varied according to the operational methods. The prevalence of class II sarcopenia, which generally indicates a population with low muscle mass, was $9.3 \%$ in men and $0.2 \%$ in women, respectively, when defined by the ASM $/ \mathrm{ht}^{2}$ index. It was around $10 \%$ in both men and women by the ASM/wt index. However, it increased to $26.8 \%$ in men and $27.9 \%$ in women, respectively, when the ASM/BMI index was used (Fig. 2A).

The increasing patterns in sarcopenia prevalence with aging are commonly observed with all three indices. However, the rates are more than twice as great when the ASM/BMI index is used compared to ASM/ht ${ }^{2}$ in the same age groups (Fig. 2B). Similarly, the sarcopenia prevalence varied according the operational methods in women: the rates of class II sarcopenia were less than ${ }_{1} \%$ by the ASM $/ \mathrm{ht}^{2}$ method, around $10 \%$ by the ASM/wt index, and $20 \%$ to $36 \%$ by the ASM/BMI index (Fig. 2 C). Increasing trends in sarcopenia prevalence with aging were observed using the ASM/ht ${ }^{2}$ or ASM/BMI methods, but not when using the ASM/wt index (Fig. 2C).

These three operational methods have been recommended for assessing muscle mass by international study groups on sarcopenia. However, notable differences are observed in the results obtained by practical applications of each method. Thus, the determination of which operational methods are most appropriate for determining the degree of low muscle mass that actually contributes to weakness and slowness in given individuals remains inconclusive.

\section{CONCLUSIONS}

Sarcopenia is defined by a composite of muscle mass, muscle strength, and physical performance. Low muscle mass potentially contributes to disability and frailty in the elderly. Recently, sarcopenia has attracted sub- 
stantial attention because of its potential relationship with diverse adverse health outcomes in elderly people including falling, fractures, infections, cardiovascular risks, metabolic impairments, and ultimately, mortality.

The topics of recent studies on sarcopenia are now moving from conceptual definitions to practical definitions that may be used in practice as well as in research. In this context, several different operational methods, including ASM/ht ${ }^{2}$, ASM/wt, and ASM/BMI, have been sequentially proposed since 1998. International study groups for sarcopenia have released consensus guidelines about the concept, definition, and diagnosis of sarcopenia using the skeletal muscle mass indices. Recently, many studies on the prevalence of sarcopenia have been published in different countries. However, the operational methods for evaluating muscle mass in these studies have differed. Accordingly, the prevalence of sarcopenia and its relationship to clinical outcomes vary widely depending on the methods used to define low lean mass. Therefore, there is a need to elucidate which operational definition is ideal for identifying sarcopenic subjects at high risk. Large-scale prospective studies to address this issue are urgently warranted. A collaborative study merging many cohorts should be initiated to refine and standardize the working definition of sarcopenia and to establish this condition as a significant predictor of adverse health outcomes.

\section{Conflict of interest}

No potential conflict of interest relevant to this article was reported.

\section{Acknowledgments}

This research was supported by a Basic Science Research Program through the National Research Foundation of Korea (NRF) funded by the Ministry of Science, ICT \& Future Planning (NRF-2014R1A1A3051310) to KMK and by research grants from the Seoul National University Bundang Hospital to SL (06-2013-202).

\section{REFERENCES}

1. Frontera WR, Ochala J. Skeletal muscle: a brief review of structure and function. Calcif Tissue Int 2015;96:183-195.

2. Lee SW, Youm Y, Lee WJ, et al. Appendicular skeletal muscle mass and insulin resistance in an elderly Korean population: the Korean social life, health and aging project-health examination cohort. Diabetes Metab J 2015;39:37-45.

3. Hughes VA, Frontera WR, Wood M, et al. Longitudinal muscle strength changes in older adults: influence of muscle mass, physical activity, and health. J Gerontol A Biol Sci Med Sci 2001;56:B209-B217.

4. Cheng Q, Zhu X, Zhang X, et al. A cross-sectional study of loss of muscle mass corresponding to sarcopenia in healthy Chinese men and women: reference values, prevalence, and association with bone mass. J Bone Miner Metab 2014;32:78-88.

5. Fielding RA, Vellas B, Evans WJ, et al. Sarcopenia: an undiagnosed condition in older adults. Current consensus definition: prevalence, etiology, and consequences. International Working Group on Sarcopenia. J Am Med Dir Assoc 2011;12:249-256.

6. Choi KM. Sarcopenia and sarcopenic obesity. Endocrinol Metab (Seoul) 2013;28:86-89.

7. Kim HT, Kim HJ, Ahn HY, Hong YH. An analysis of age-related loss of skeletal muscle mass and its significance on osteoarthritis in a Korean population. Korean J Intern Med 2016;31:585-593.

8. Ochi M, Kohara K, Tabara Y, et al. Arterial stiffness is associated with low thigh muscle mass in middle-aged to elderly men. Atherosclerosis 2010;212:327-332.

9. He H, Liu Y, Tian Q, Papasian CJ, Hu T, Deng HW. Relationship of sarcopenia and body composition with osteoporosis. Osteoporos Int 2016;27:473-482.

10. Ahima RS, Park HK. Connecting myokines and metabolism. Endocrinol Metab (Seoul) 2015;30:235-245.

11. Chin SO, Rhee SY, Chon S, et al. Sarcopenia is independently associated with cardiovascular disease in older Korean adults: the Korea National Health and Nutrition Examination Survey (KNHANES) from 2009. PLoS One 2013;8:e60119.

12. Atkins JL, Whincup PH, Morris RW, Lennon LT, Papacosta O, Wannamethee SG. Sarcopenic obesity and risk of cardiovascular disease and mortality: a population-based cohort study of older men. J Am Geriatr Soc 2014;62:253260.

13. Moon JS, Yoon JS, Won KC, Lee HW. The role of skeletal muscle in development of nonalcoholic fatty liver disease. Diabetes Metab J 2013;37:278-285.

14. Cruz-Jentoft AJ, Baeyens JP, Bauer JM, et al. Sarcopenia: 
European consensus on definition and diagnosis: report of the European Working Group on Sarcopenia in Older People. Age Ageing 2010;39:412-423.

15. Dam TT, Peters KW, Fragala M, et al. An evidence-based comparison of operational criteria for the presence of sarcopenia. J Gerontol A Biol Sci Med Sci 2014;69:584-590.

16. Kim JH, Lim S, Choi SH, et al. Sarcopenia: an independent predictor of mortality in community-dwelling older Korean men. J Gerontol A Biol Sci Med Sci 2014;69:12441252.

17. Cawthon PM, Peters KW, Shardell MD, et al. Cutpoints for low appendicular lean mass that identify older adults with clinically significant weakness. J Gerontol A Biol Sci Med Sci 2014;69:567-575.

18. Baumgartner RN, Koehler KM, Gallagher D, et al. Epidemiology of sarcopenia among the elderly in New Mexico. Am J Epidemiol 1998;147:755-763.

19. Janssen I, Heymsfield SB, Ross R. Low relative skeletal muscle mass (sarcopenia) in older persons is associated with functional impairment and physical disability. J Am Geriatr Soc 2002;50:889-896.

20. Chen LK, Liu LK, Woo J, et al. Sarcopenia in Asia: consensus report of the Asian Working Group for Sarcopenia. J Am Med Dir Assoc 2014;15:95-101.

21. Kim KM, Lim S, Choi SH, et al. Cardiometabolic implication of sarcopenia: The Korea National Health and Nutrition Examination Study (KNHANES) 2008-2010. IJC Metab Endocr 2014;4:63-69.

22. Bischoff-Ferrari HA, Orav JE, Kanis JA, et al. Comparative performance of current definitions of sarcopenia against the prospective incidence of falls among community-dwelling seniors age 65 and older. Osteoporos Int 2015;26:2793-2802.

23. Yoshida D, Shimada H, Park H, et al. Development of an equation for estimating appendicular skeletal muscle mass in Japanese older adults using bioelectrical impedance analysis. Geriatr Gerontol Int 2014;14:851-857.

24. Kim JH, Choi SH, Lim S, et al. Assessment of appendicular skeletal muscle mass by bioimpedance in older community-dwelling Korean adults. Arch Gerontol Geriatr 2014;58:303-307.

25. Gallagher D, Visser M, De Meersman RE, et al. Appendicular skeletal muscle mass: effects of age, gender, and ethnicity. J Appl Physiol (1985) 1997;83:229-239.

26. Sanada K, Miyachi M, Tanimoto M, et al. A cross-sectional study of sarcopenia in Japanese men and women: reference values and association with cardiovascular risk factors. Eur J Appl Physiol 2010;110:57-65.

27. Frisoli A Jr, Chaves PH, Ingham SJ, Fried LP. Severe osteopenia and osteoporosis, sarcopenia, and frailty status in community-dwelling older women: results from the Women's Health and Aging Study (WHAS) II. Bone 2011;48:952-957.

28. Looker AC. Dysmobility syndrome and mortality risk in US men and women age 50 years and older. Osteoporos Int 2015;26:93-102.

29. Newman AB, Kupelian V, Visser M, et al. Sarcopenia: alternative definitions and associations with lower extremity function. J Am Geriatr Soc 2003;51:1602-1609.

30. Lim S, Kim JH, Yoon JW, et al. Sarcopenic obesity: prevalence and association with metabolic syndrome in the Korean Longitudinal Study on Health and Aging (KLoSHA). Diabetes Care 2010;33:1652-1654.

31. Kim MK, Baek KH, Song KH, et al. Vitamin D deficiency is associated with sarcopenia in older Koreans, regardless of obesity: the Fourth Korea National Health and Nutrition Examination Surveys (KNHANES IV) 2009. J Clin Endocrinol Metab 2011;96:3250-3256.

32. Woo J, Leung J, Morley JE. Defining sarcopenia in terms of incident adverse outcomes. J Am Med Dir Assoc 2015;16:247-252.

33. Tyrovolas S, Koyanagi A, Olaya B, et al. The role of muscle mass and body fat on disability among older adults: a cross-national analysis. Exp Gerontol 2015;69:27-35.

34. Studenski SA, Peters KW, Alley DE, et al. The FNIH sarcopenia project: rationale, study description, conference recommendations, and final estimates. J Gerontol A Biol Sci Med Sci 2014;69:547-558.

35. Kim TN, Park MS, Lim KI, et al. Relationships between sarcopenic obesity and insulin resistance, inflammation, and vitamin D status: the Korean Sarcopenic Obesity Study. Clin Endocrinol (Oxf) 2013;78:525-532.

36. Srikanthan P, Karlamangla AS. Relative muscle mass is inversely associated with insulin resistance and prediabetes: findings from the third National Health and Nutrition Examination Survey. J Clin Endocrinol Metab 2011;96:2898-2903.

37. Meng NH, Li CI, Liu CS, et al. Comparison of height- and weight-adjusted sarcopenia in a Taiwanese metropolitan older population. Geriatr Gerontol Int 2015;15:45-53.

38. Bijlsma AY, Meskers CG, van Heemst D, Westendorp RG, de Craen AJ, Maier AB. Diagnostic criteria for sarcope- 
nia relate differently to insulin resistance. Age (Dordr) 2013;35:2367-2375.

39. Scott D, Hayes A, Sanders KM, Aitken D, Ebeling PR, Jones G. Operational definitions of sarcopenia and their associations with 5-year changes in falls risk in community-dwelling middle-aged and older adults. Osteoporos Int 2014;25:187-193.
40. Batsis JA, Barre LK, Mackenzie TA, Pratt SI, Lopez-Jimenez F, Bartels SJ. Variation in the prevalence of sarcopenia and sarcopenic obesity in older adults associated with different research definitions: dual-energy X-ray absorptiometry data from the National Health and Nutrition Examination Survey 1999-2004. J Am Geriatr Soc 2013;61:974980. 